\title{
Neural correlates underlying naloxone-induced amelioration of sexual behavior deterioration due to an alarm pheromone
}

\author{
Tatsuya Kobayashi ${ }^{+}$, Yasushi Kiyokawa *, Yukari Takeuchi and Yuji Mori \\ Laboratory of Veterinary Ethology, Graduate School of Agricultural and Life Sciences, The University of Tokyo, Bunkyo, Tokyo, Japan
}

Edited by:

Xiaogang Wu, Institute for Systems

Biology, USA

Reviewed by:

Tianxiao Huan, Framingham Heart

Study, USA

Socorro Retana-Marquez,

Universidad Autónoma

Metropolitana, Mexico

\section{${ }^{*}$ Correspondence:}

Yasushi Kiyokawa, Laboratory of

Veterinary Ethology, Graduate

School of Agricultural and Life

Sciences, The University of Tokyo,

1-1-1 Yayoi, Bunkyo-ku, Tokyo

113-8657, Japan

e-mail: akiyo@mail.ecc.u-tokyo.ac.jp

${ }^{\dagger}$ Present address:

Tatsuya Kobayashi, Product Science

Division, Japan Tobacco Inc., 6-2,

Umegaoka, Aoba-ku, Yokohama,

Kanagawa 227-8512, Japan
Sexual behavior is suppressed by various types of stressors. We previously demonstrated that an alarm pheromone released by stressed male Wistar rats is a stressor to other rats, increases the number of mounts needed for ejaculation, and decreases the hit rate (described as the number of intromissions/sum of the mounts and intromissions). This deterioration in sexual behavior was ameliorated by pretreatment with the opioid receptor antagonist naloxone. However, the neural mechanism underlying this remains to be elucidated. Here, we examined Fos expression in 31 brain regions of pheromone-exposed rats and naloxone-pretreated pheromone-exposed rats $60 \mathrm{~min}$ after 10 intromissions. As previously reported, the alarm pheromone increased the number of mounts and decreased the hit rate. In addition, Fos expression was increases in the anterior medial division (BNSTam), anterior lateral division (BNSTal) and posterior division (BNSTp) of the bed nucleus of the stria terminalis, parvocellular part of the paraventricular nucleus of the hypothalamus, arcuate nucleus, dorsolateral and ventrolateral periaqueductal gray, and nucleus paragigantocellularis (nPGi). Fos expression was decreased in the magnocellular part of the paraventricular nucleus of the hypothalamus. Pretreatment with naloxone blocked the pheromone-induced changes in Fos expression in the magnocellular part of the paraventricular nucleus of the hypothalamus, ventrolateral periaqueductal gray, and nPGi. Based on these results, we hypothesize that the alarm pheromone deteriorated sexual behavior by activating the ventrolateral periaqueductal gray-nucleus paragigantocellularis cluster and suppressing the magnocellular part of the paraventricular nucleus of the hypothalamus (PVN) via the opioidergic pathway.

Keywords: opioid, alarm pheromone, sexual behavior, nucleus paragigantocellularis, periaqueductal gray, paraventricular nucleus of hypothalamus

\section{INTRODUCTION}

Sexual behavior is very important for reproduction in animals. It can be suppressed by various types of manipulations; for example, social isolation (Barrot et al., 2005), maternal separation (Rhees et al., 2001), prenatal stress (Wang et al., 2006), parasitic infection (Lin et al., 1990; Klein, 2003), immobilization, foot shock, and water immersion (Retana-Marquez et al., 1996) suppress male sexual behavior in rodents. In addition, we have found that an alarm pheromone released from stressed conspecifics deteriorates male sexual behavior in rats (Kobayashi et al., 2011).

It is well-known that stressed animals release specific odors, possibly to warn or alarm nearby conspecifics, some of which are defined as alarm pheromones (Karlson and Luscher, 1959; Inagaki et al., 2014). We previously investigated an alarm pheromone that causes an increase in body temperature in rats placed in a box, which recently placed two male rats that had received foot shocks (Kikusui et al., 2001). This phenomenon suggests that the rats that received the foot shocks served as pheromone donors and released an alarm pheromone when they received the shocks. It is possible to induce pheromone release by applying electrical stimulation to the perianal region of an anesthetized donor (Kiyokawa et al., 2004); therefore, we established a method that traps this pheromone in water (Kiyokawa et al., 2005a). We found that this pheromone-containing water can evoke a variety of stress responses, depending on the experimental model (Kiyokawa et al., 2006; Inagaki et al., 2008; Kobayashi et al., 2011), through the vomeronasal system (Kiyokawa et al., 2007, 2013a) and main olfactory system (Inagaki et al., 2014).

This alarm pheromone is a suitable candidate to analyze the effects of stress on sexual behavior because the natural stress responses were induced through the olfactory system. We observed the sexual behavior of pairs of rats in the presence of this pheromone and found that it increases the number of mounts and decreases the hit rate in male rats (Kobayashi et al., 2011). Subsequently, we found that this pheromone-induced deterioration in sexual behavior is gender-specific because it occurs when the pheromone was presented to the male, but not the female, before a sexual encounter (Kobayashi et al., 2011). We conducted Fos mapping in a variety of brain regions and found that the nucleus paragigantocellularis (nPGi) is a key region 
associated with the pheromone-induced deterioration. We have found increased Fos expression in the anterior medial division (BNSTam), anterior lateral division (BNSTal), and posterior division (BNSTp) of the bed nucleus of the stria terminalis, medial and basal amygdala, and corticotropin-releasing hormone $(\mathrm{CRH})$-containing neurons in the paraventricular nucleus of the hypothalamus (PVN), which are in the parvocellular part of the PVN (pPVN) (Kobayashi et al., 2013a). However, we did not find any other candidate nuclei that were directly involved in this deterioration or that activated the nPGi. This failure might be attributed to neural activation from ejaculation, which obscured Fos expression associated with pheromone-induced sexual behavior deterioration.

In parallel with the immunohistochemical analyses, we pharmacologically analyzed the neural mechanism of deterioration. We found that systemic pretreatment with the opioid receptor antagonist naloxone attenuated sexual deterioration in male rats. This suggests that opioids are involved in the neural mechanism (Kobayashi et al., 2013b). However, regions in which we found increased Fos expression during deterioration (Kobayashi et al., 2013a), such as the nPGi and several other nuclei, to our knowledge, do not express opioid receptors. Therefore, it remains unclear how opioids are involved in the deterioration of sexual behavior by the alarm pheromone.

To address these issues, in this study, we measured Fos expression in 31 brain regions in pheromone-exposed rats that showed a deterioration of sexual behavior but did not achieve ejaculation. We also assessed pheromone-exposed rats that were pretreated with naloxone in order to obtain further information about the role of opioids in deterioration.

\section{MATERIAL AND METHODS ANIMALS}

Twenty-five sexually naïve male (aged 7.5 weeks) and female (aged 8.5 weeks) Wistar Imamichi rats were used in this study (Institute for Animal Reproduction, Ibaraki, Japan). All animals were provided with food and water ad libitum and kept on a 12-h light-dark cycle (lights turned off at 2000). The colony room was maintained at a constant temperature $\left(24 \pm 1^{\circ} \mathrm{C}\right)$ and humidity $(40-45 \%)$. Animals of the same sex were housed in pairs in wire-topped, transparent cages $(410 \times 250 \times 180 \mathrm{~mm})$ with wood shavings for bedding. All procedures were approved by the Animal Care and Use Committee of the Faculty of Agriculture of The University of Tokyo.

\section{PREPARATION OF THE ALARM PHEROMONE}

Before the sexual behavior test, water samples that contained either the alarm pheromone or a control odor were prepared according to a previously described method (Kiyokawa et al., 2005a; Kobayashi et al., 2013b). Adult male Wistar Imamichi rats (aged 12-16 weeks) were anesthetized and 2 intradermal needles $(27 \mathrm{G})$ were attached at either the neck or perianal region. After spraying purified water $(5 \mathrm{~mL})$ on the ceiling of an acrylic box $(200 \times 200 \times 100 \mathrm{~mm})$, the anesthetized donor was placed in the box and given 15 electrical stimuli ( $10 \mathrm{~V}$ for $1 \mathrm{~s}$ ) through the needles for $5 \mathrm{~min}$ at $20 \mathrm{~s}$ intervals. Care was taken that water droplets did not fall from the ceiling and that the donor was kept under anesthesia during the stimulations. The electrical stimulation of the perianal region induced the release of alarm pheromones and stimulation of the neck region was conducted to provide a similar amount of control olfactory stimuli. Following this, the donor was removed and the water droplets containing the alarm pheromone or control odor were collected. The samples were stored at $4^{\circ} \mathrm{C}$ until use.

\section{SEXUAL BEHAVIOR TEST}

Sexual behavior tests were conducted as described in previous studies (Kobayashi et al., 2011, 2013a,b). Briefly, 1 day before the test, male rats were housed individually and acclimatized for $30 \mathrm{~min}$ to the experimental room and devices. Female rats were also acclimatized to the experimental room for $30 \mathrm{~min}$. On the test day, the test was conducted in the home cage of the male subject that had been placed in the experimental room under a dim red light. Saline or naloxone $\left(40 \mathrm{mg} \cdot \mathrm{kg}^{-1}\right.$ dissolved in saline; SigmaAldrich, St. Louis, MO) was administered intraperitoneally to the male $60 \mathrm{~min}$ before the test. At the beginning of the test, pheromone or control samples $(750 \mu \mathrm{L}$ each) were dropped on two sheets of filter paper $(5 \times 5 \mathrm{~cm})$ that were placed in acrylic plates $(12 \times 6 \mathrm{~cm}, 3 \mathrm{~mm}$ thickness $)$, one of which had 18 holes, $9 \mathrm{~mm}$ in diameter. The plates were then placed in the home cage for $5 \mathrm{~min}$. Following this, the plates were removed and the female was introduced. Sexual behavior was video recorded and observed in an adjacent room during the test. When the tenth intromission was observed, the female was removed. Twenty-five male rats were divided into three groups according to the type of sample and injection: control odor and saline injection (Control-SAL; $n=8$ ), alarm pheromone and saline injection (Pheromone-SAL; $n=8$ ), and alarm pheromone and naloxone injection (Pheromone-NAL; $n=9)$.

\section{IMMUNOHISTOCHEMISTRY}

Immunohistochemistry was conducted as described in previous studies (Kiyokawa et al., 2013b, 2014; Kobayashi et al., 2013a). Briefly, $60 \mathrm{~min}$ after the tenth intromission, male rats were deeply anesthetized with sodium pentobarbital and perfused intracardially with $0.9 \%$ saline followed by $4 \%$ paraformaldehyde in $0.1 \mathrm{M}$ phosphate buffer. The brain was removed and immersed overnight in the same fixative and then placed in $30 \%$ sucrose in phosphate buffer for cryoprotection. The brain was cut into $25-\mu \mathrm{m}$-thick coronal sections. Six successive sections containing some of the target regions were collected, and the second and fifth sections were stained with Cresyl violet to confirm localization in the brain. The remaining sections were incubated in $\mathrm{H}_{2} \mathrm{O}_{2}$, then primary antibody targeting the Fos protein (PC38, Merck Millipore, Billerica, MA) for $65 \mathrm{~h}$, and biotinylated anti-rabbit secondary antibody (BA-1000, Vector Laboratories, Burlingame, CA) for $2 \mathrm{~h}$. Following this, the sections were processed using the $\mathrm{ABC}$ kit (Vector Laboratories) and developed using a 3,3'-diaminobenzidine solution with nickel intensification.

\section{QUANTIFICATION}

Fos expression was analyzed as described in previous studies (Kiyokawa et al., 2005b, 2013b; Kobayashi et al., 2013a). Four 
sections showing each of the 31 regions were imaged using a microscope equipped with a digital camera (DP30BW; Olympus, Tokyo, Japan). The number of c-Fos-immunoreactive cells in a $0.5-\mathrm{mm}$ square was counted bilaterally using Image J software (version 1.41) by an experimenter who was blind to the experimental groups. The raphe nuclei are located in the center of the brain; therefore, Fos expression could not be counted bilaterally. In some cases, the designated area to be counted was smaller than the boundaries of the $0.5-\mathrm{mm}$ square; therefore, only the cells in the brain region of interest, and not in other regions within the square, were counted.

\section{DATA ANALYSIS AND STATISTICAL PROCEDURES}

Male sexual behavior was analyzed as described in previous studies by a researcher who was blind to the experimental conditions (Kobayashi et al., 2011, 2013a,b). The following measures of sexual behavior were recorded: mount latency (pelvic thrusting from the rear of the female rat without penile insertion), intromission latency (deeper pelvic thrusting from the rear of the female rat with penile insertion), latency for tenth intromission (time from the first intromission to tenth intromission), and number of mounts (number of mounts needed for 10 intromissions). In addition, the hit rate was calculated. Behavioral and immunohistochemical data are expressed as mean \pm the standard error of the mean (SEM). The significance was set at $P<0.05$ for all statistical tests. All data were analyzed by one-way analysis of variance followed by Fisher's PLSD post-hoc test.

\section{RESULTS}

The number of mounts $\left[F_{(2,22)}=5.47, P=0.012\right.$; Figure 1A $]$ and hit rate $\left[F_{(2,22)}=5.40, P=0.012\right.$; Figure $\left.1 \mathrm{~B}\right]$ were significantly different between the groups, whereas the mount latency $\left[F_{(2,22)}=0.11, \quad P=0.892 ;\right.$ Figure 1C $]$, intromission latency $\left[F_{(2,22)}=0.68, P=0.519\right.$; Figure 1D], and latency to tenth intromission $\left[F_{(2,22)}=0.30, P=0.746\right.$; Figure 1E $]$ were not. Post-hoc tests revealed that the Pheromone-SAL group showed an increased number of mounts $(P=0.017)$ and decreased hit rate $(P=0.022)$ when compared with the Control-SAL group. However, these measures were not different between the ControlSAL and Pheromone-NAL groups (number of mounts, $P=$ 0.653 ; hit rate, $P=0.565$ ). A post-hoc test also confirmed that the Pheromone-SAL group showed an increased number of mounts $(P=0.005)$ and decreased hit rate $(P=0.005)$ when compared with the Pheromone-NAL group.

We measured Fos expression in 31 brain regions (Figure 2). The mean number of c-Fos-immunoreactive cells is summarized
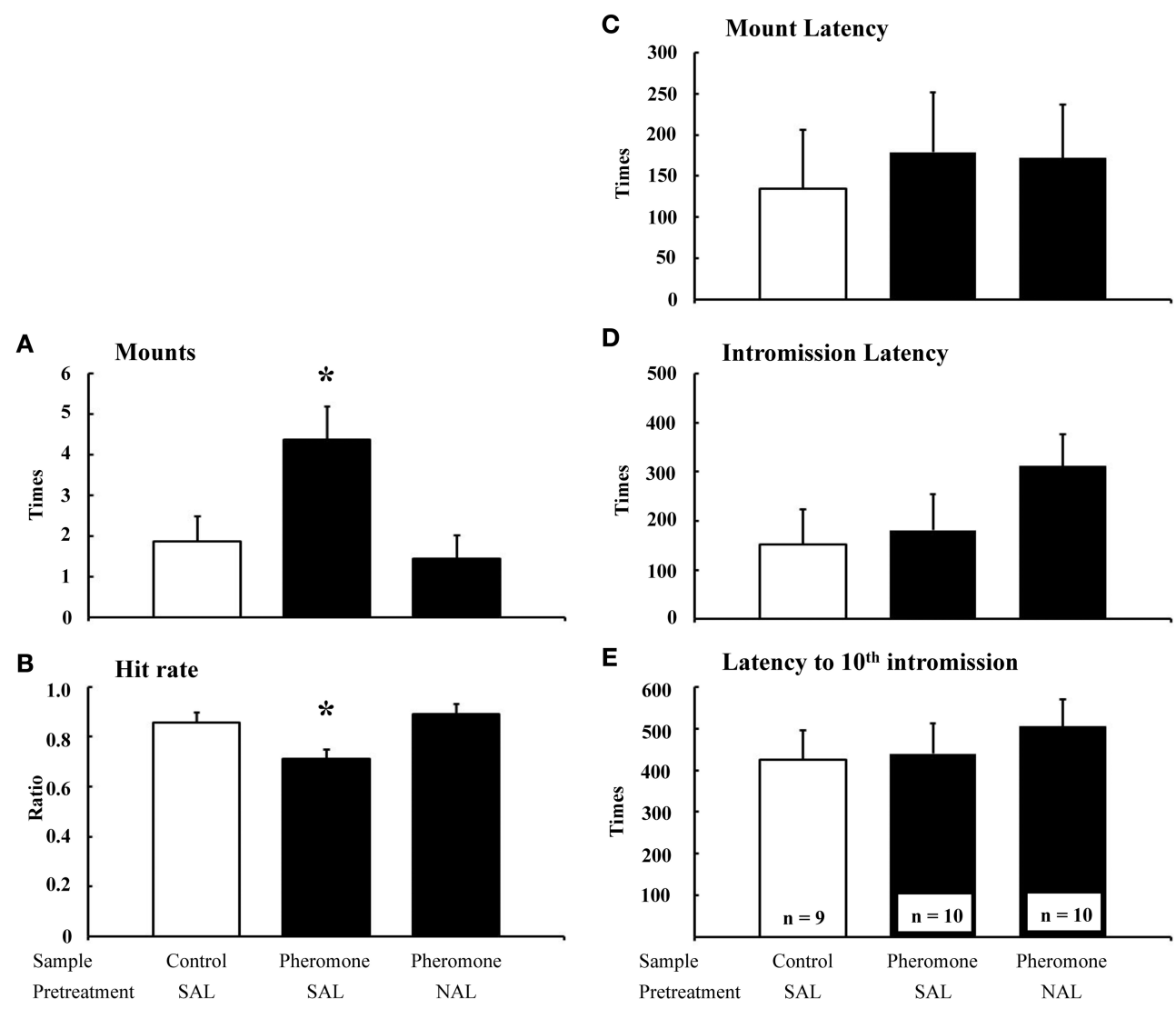

FIGURE 1 | The effects of naloxone on the pheromone-induced deterioration of sexual behavior. Number of mounts (A), hit rate (B) mount latency (C), intromission latency (D), and latency to 10th intromission (E) of the subjects (mean + SEM). Subjects were pretreated

with either saline $(\mathrm{SAL})$ or naloxone $\left(40 \mathrm{mg} \cdot \mathrm{kg}^{-1} ; \mathrm{NAL}\right)$ and exposed to a control odor (Control) or an alarm pheromone (Pheromone). ${ }^{*} P<0.05$ compared with the Control-SAL group, ANOVA followed by Fisher's PLSD post-hoc test. 


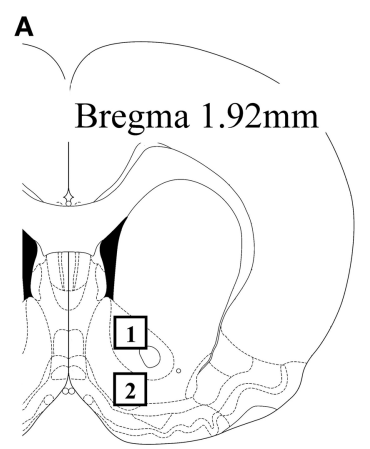

B

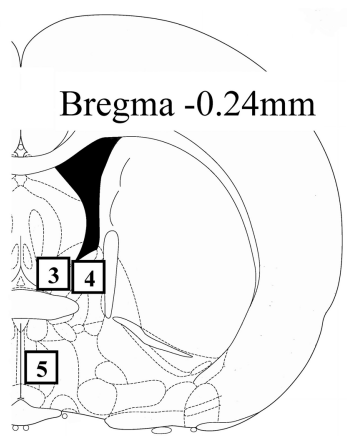

D
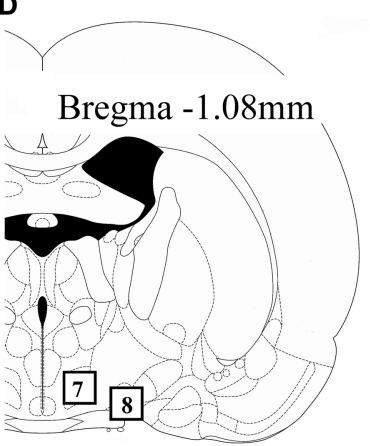

G

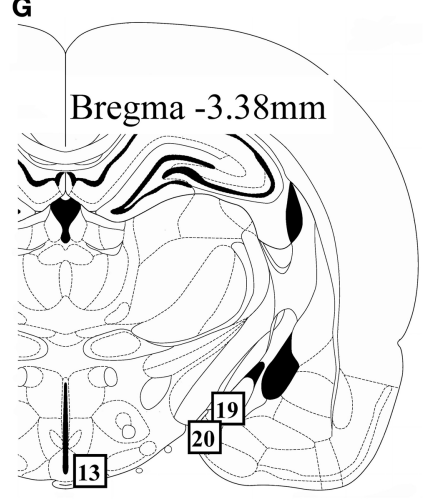

$\mathbf{J}$

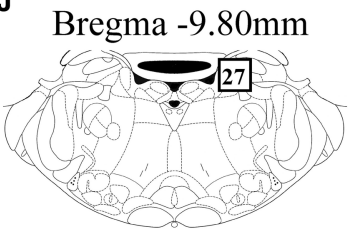

E
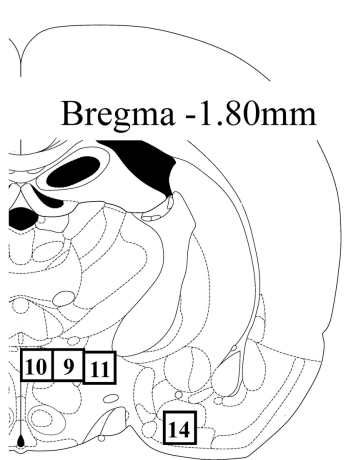

C

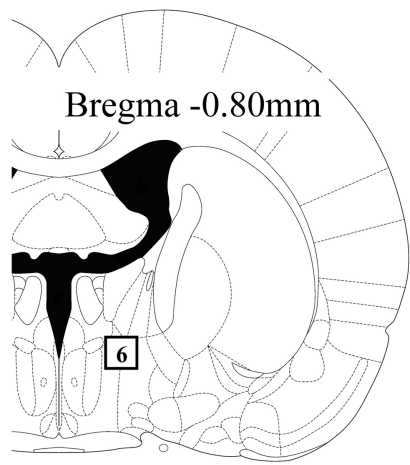

$\mathbf{F}$

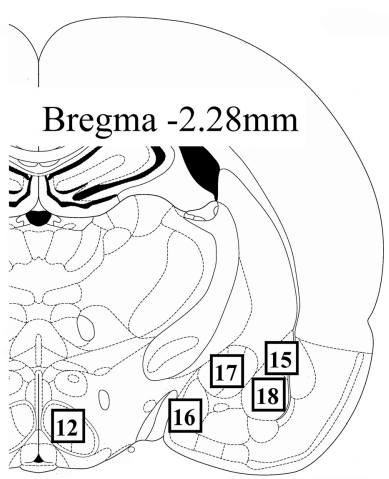

H

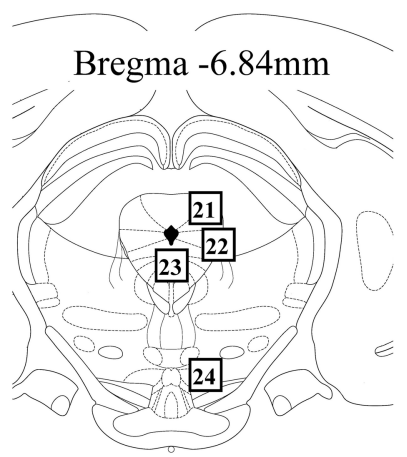

I Bregma $-8.40 \mathrm{~mm}$

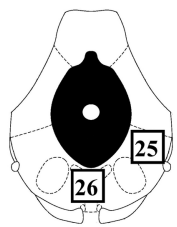

FIGURE 2 | Schematic diagrams showing the location of brain regions (open square containing numbers) in which c-Fos-immunoreactive cells were counted. The regions analyzed were: the nucleus accumbens core and shell (A), BNSTam, BNSTal, and medial preoptic area (B), BNSTp (C), lateral hypothalamic area and supraoptic nucleus (D), mPVN, pPVN, peduncular lateral hypothalamus, and anterior cortical amygdala (E), ventromedial nucleus of hypothalamus and lateral, anterior medial, central, and basolateral amygdala (F), Arc and posterodorsal and posteroventral medial amygdala (G), dIPAG, lateral periaqueductal gray, dorsal raphe nucleus, and ventral tegmental area (H), vIPAG and dorsal region of the dorsal raphe nucleus (I), locus coeruleus (J), nPGi and raphe magnus and pallidus (K), and raphe obscurus (L). This figure was adapted from a rat brain atlas (Paxinos and Watson, 2007). For an explanation of the abbreviations, see the corresponding numbers in Table $\mathbf{1}$. in Table 1. Fos expression was significantly different among the groups in the nucleus accumbens core $\left[F_{(2,22)}=8.21\right.$, $P=0.002]$ and shell $\left[F_{(2,22)}=6.07, P=0.008\right]$, BNSTam $\left[F_{(2,22)}=4.81, P=0.019\right]$, BNSTal $\left[F_{(2,22)}=12.9, P<0.001\right]$,
BNSTp $\left[F_{(2,22)}=6.66, \quad P=0.006\right]$, magnocellular part of the PVN [mPVN; $\left.F_{(2,22)}=8.09, \quad P=0.002\right], \quad \mathrm{pPVN}$ $\left[F_{(2,22)}=8.28, P=0.002\right]$, ventromedial nucleus of hypothalamus $\left[F_{(2,22)}=5.55, \quad P=0.011\right]$, arcuate nucleus [Arc; 
Table 1 | The number of c-Fos-immunoreactive cells per $0.25 \mathrm{~mm}^{2}$ in various brain regions.

\begin{tabular}{|c|c|c|c|}
\hline Regions & Control-SAL (8) & Pheromone-SAL (8) & Pheromone-NAL (9) \\
\hline 9. Magnocellular part of the paraventricular nucleus of hypothalamus (mPVN) & $17.4 \pm 2.0$ & $9.8 \pm 1.0 * P=0.003$ & $17.9 \pm 1.6$ \\
\hline 28. Nucleus paragigantocellularis (nPGi) & $7.3 \pm 0.8$ & $21.5 \pm 2.6^{*} P<0.001$ & $P=0.226$ \\
\hline \multicolumn{4}{|c|}{ THE REGIONS WHERE PRETREATMENT WITH NALOXONE DID NOT BLOCK THE ACTIVATION BY THE PHEROMONE } \\
\hline 6. Posterior division of the bed nucleus of the stria terminalis (BNSTp) & $19.9 \pm 2.3$ & $37.7 \pm 5.9 * P=0.004$ & $36.3 \pm 2.3^{*} \quad P=0.005$ \\
\hline 10. Parvocellular part of the paraventricular nucleus of hypothalamus (pPVN) & $34.6 \pm 3.0$ & $51.6 \pm 4.8 * P=0.036$ & $64.7 \pm 6.7^{*} \quad P<0.001$ \\
\hline 13. Arcuate nucleus (Arc) & $9.4 \pm 1.9$ & $30.3 \pm 4.7^{*} P=0.001$ & $34.6 \pm 4.1 * \quad P<0.001$ \\
\hline 21. Dorsolateral periaqueductal gray (dIPAG) & $16.2 \pm 1.6$ & $23.6 \pm 1.8 * P=0.007$ & $22.9 \pm 1.7^{*} \quad P=0.011$ \\
\hline \multicolumn{4}{|c|}{ THE REGIONS WHERE PRETREATMENT WITH NALOXONE, BUT NOT PHEROMONE EXPOSURE, CAUSED ACTIVATION } \\
\hline 23. Dorsal raphe nucleus & $6.0 \pm 0.8$ & $6.3 \pm 1.1 \quad P=0.813$ & $10.0 \pm 0.8^{*} \quad P=0.005$ \\
\hline \multicolumn{4}{|c|}{ THE REGIONS WHERE NEITHER THE PHEROMONE NOR PRETREATMENT WITH NALOXONE CAUSED ACTIVATION } \\
\hline 5. Medial preoptic area & $81.9 \pm 7.7$ & $90.6 \pm 6.2$ & $88.8 \pm 3.9$ \\
\hline 7. Lateral hypothalamic area & $13.9 \pm 2.9$ & $17.8 \pm 2.5$ & $19.6 \pm 2.8$ \\
\hline 8. Supraoptic nucleus & $13.8 \pm 3.2$ & $13.5 \pm 2.6$ & $8.9 \pm 0.9$ \\
\hline 11. Peduncular lateral hypothalamus & $16.1 \pm 3.1$ & $20.2 \pm 2.4$ & $25.6 \pm 2.9$ \\
\hline 14. Anterior cortical amygdala & $36.5 \pm 4.1$ & $34.6 \pm 5.2$ & $34.2 \pm 4.0$ \\
\hline 15. Lateral amygdala & $6.5 \pm 1.0$ & $6.8 \pm 1.1$ & $7.7 \pm 0.5$ \\
\hline 16. Anterior medial amygdala & $24.0 \pm 3.1$ & $32.4 \pm 3.3$ & $31.9 \pm 2.8$ \\
\hline 18. Basal amygdala & $13.6 \pm 2.0$ & $12.3 \pm 2.6$ & $14.0 \pm 2.3$ \\
\hline 30. Raphe pallidus & $4.9 \pm 0.5$ & $5.0 \pm 0.4$ & $5.5 \pm 1.0$ \\
\hline 31. Raphe obscurus & $11.9 \pm 2.7$ & $12.2 \pm 2.8$ & $18.1 \pm 2.1$ \\
\hline
\end{tabular}

Data are expressed as means \pm SEM. For the location of each region, see the corresponding numbers in Figure 2 . The number of rats per group is given in parentheses. ${ }^{*} P<0.05$ compared to the Control-SAL group, One-Way ANOVA followed by Fisher's PLSD post-hoc test.

$F(2,22)=12.3, P<0.001]$, central amygdala $\left[F_{(2,22)}=16.0\right.$, $P<0.001$ ], dorsolateral [dlPAG; $F_{(2,22)}=5.53, P=0.011$ ] and ventrolateral periaqueductal gray $\left[\mathrm{vlPAG} ; F_{(2,22)}=28.0\right.$, $P<0.001]$, dorsal raphe nucleus $\left[F_{(2,22)}=6.11, P=0.008\right]$, and $\mathrm{nPGi}\left[F_{(2,22)}=21.9, P<0.001\right]$.

Post-hoc tests revealed that these brain regions could be divided into the following 3 categories. The first category is composed of the mPVN (Figures 3A-C), vlPAG (Figures 3D-F), and nPGi (Figures 3G-I), in which pretreatment with naloxone blocked the pheromone-induced change in Fos expression. Compared with the Control-SAL group, the PheromoneSAL group $(P=0.003)$, but not the Pheromone-NAL group $(P=0.795)$, showed decreased Fos expression in the mPVN. Fos expression in the vlPAG and nPGi in the PheromoneSAL group (vlPAG, $P<0.001$; nPGi, $P<0.001$ ), but not the
Pheromone-NAL group (vlPAG, $P=0.969$; nPGi, $P=0.226$ ) was increased compared with that in the Control-SAL group. The second category is composed of the BNSTam, BNSTal, BNSTp, pPVN, Arc, and dlPAG, in which pretreatment with naloxone did not block the pheromone-induced increase Fos expression. Both the Pheromone-SAL (BNSTam, $P=0.009$; BNSTal, $P=0.012$; BNSTp, $P=0.004$; pPVN, $P=0.036$; Arc, $P=0.001$; dlPAG, $P=0.007)$ and Pheromone-NAL groups (BNSTam, $P=0.020$; BNSTal, $P<0.001$; BNSTp, $P=0.005$; pPVN, $P<0.001$; Arc, $P<0.001$; dlPAG, $P=0.011)$ showed increased Fos expression when compared with the Control-SAL group in these regions. The last category is composed of the nucleus accumbens core and shell, ventromedial nucleus of hypothalamus, central amygdala, and dorsal raphe nucleus, in which pretreatment with naloxone increased Fos expression but the alarm pheromone 


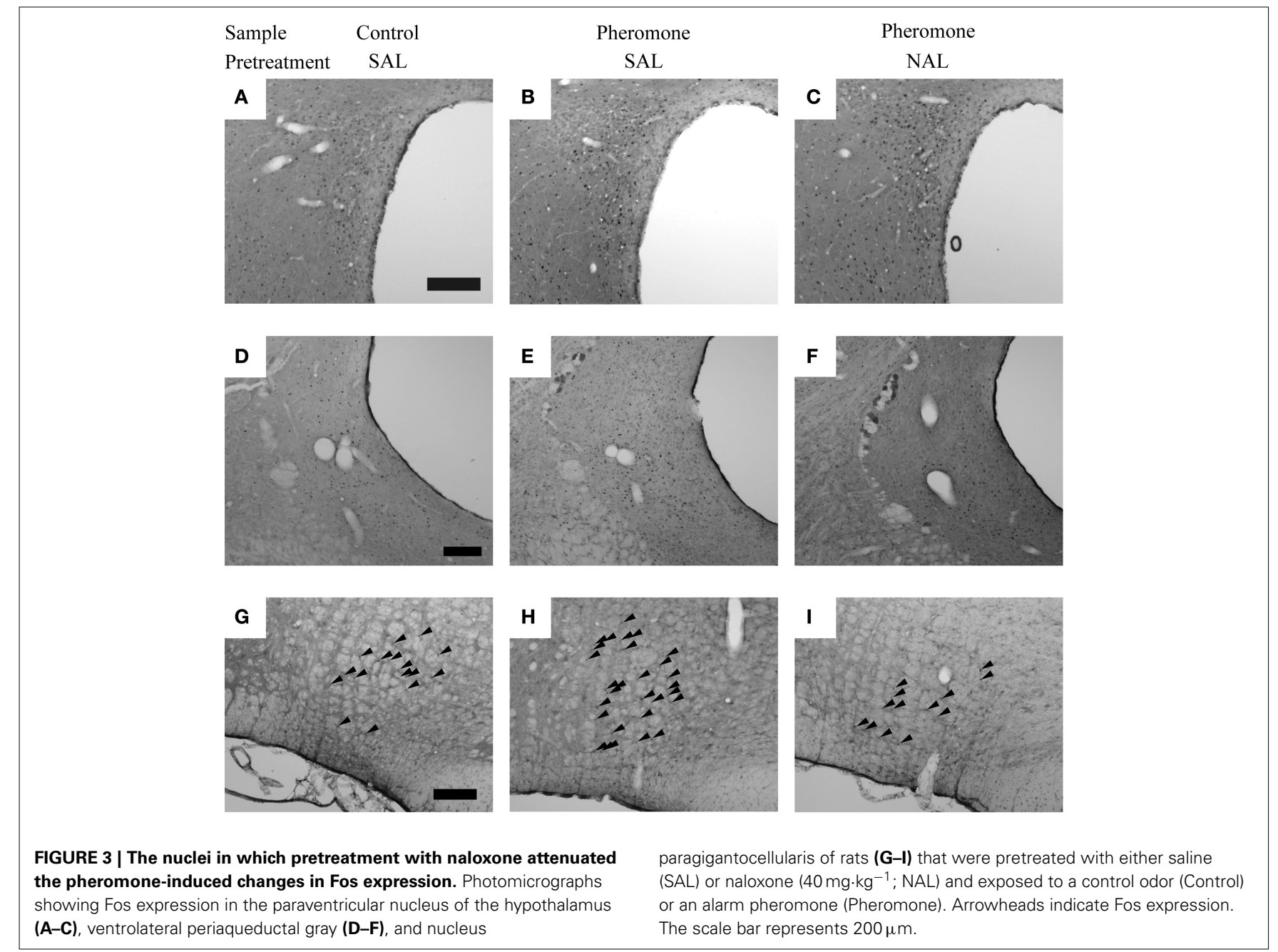

did not. In these regions, the Pheromone-NAL group (nucleus accumbens core, $P=0.001$; nucleus accumbens shell, $P=0.020$; ventromedial nucleus of hypothalamus, $P=0.005$; central amygdala, $P<0.001$; dorsal raphe nucleus, $P=0.005$ ), but not the Pheromone-SAL group (nucleus accumbens core, $P=0.470$; nucleus accumbens shell, $P=0.436$; ventromedial nucleus of hypothalamus, $P=0.568$; central amygdala, $P=0.939$; dorsal raphe nucleus, $P=0.813$ ), showed increased Fos expression compared with the Control-SAL group.

Post-hoc tests confirmed a significant difference between the Pheromone-SAL and Pheromone-NAL groups in nuclei in the first (mPVN, $P=0.001$; vlPAG, $P<0.001$; nPGi, $P<0.001$ ) and third categories (nucleus accumbens core, $P=0.006$; nucleus accumbens shell, $P=0.003$; ventromedial nucleus of hypothalamus, $P=0.020$; central amygdala, $P<0.001$; dorsal raphe nucleus, $P=0.009$ ). Similarly, Fos expression was not different between the Pheromone-SAL and Pheromone-NAL groups in nuclei in the second category (BNSTam, $P=0.665$; BNSTp, $P=0.787$; pPVN, $P=0.090 ;$ Arc, $P=0.437$; dlPAG, $P=0.774$ ), except in the BNSTal, where the Pheromone-NAL group showed increased Fos expression compared with the Pheromone-SAL group $(P=0.034)$.

\section{DISCUSSION}

In the present study, we attempted to clarify the neural mechanisms underlying the alarm pheromone-induced deterioration in sexual behavior in male rats and assessed how opioids were involved. We observed that the pheromone affected Fos expression in the BNSTam, BNSTal, BNSTp, mPVN, pPVN, Arc, dlPAG, vlPAG, and nPGi. In addition, pretreatment with naloxone blocked these effects in the mPVN, vlPAG, and nPGi. Based on these results, we have revised our hypothesis regarding the neural mechanisms underlying the alarm pheromone-induced deterioration in sexual behavior (Kobayashi et al., 2013a,b) as follows: when a male rat detects the alarm pheromone, the vomeronasal system, including the BNSTp, and the main olfactory system (Inagaki et al., 2014) receive information about the pheromone. Information from these 2 olfactory systems activates the pPVN, which subsequently activates opioidergic neurons in the Arc. The opioidergic neurons suppress the mPVN and indirectly activate the vlPAG-nPGi cluster. As a result, sexual behavior deteriorates (Figure 4).

In addition to the nuclei found in our previous study, and excluding the neural responses related to ejaculation, we found activation in the Arc, dlPAG, and vlPAG and suppression in the 


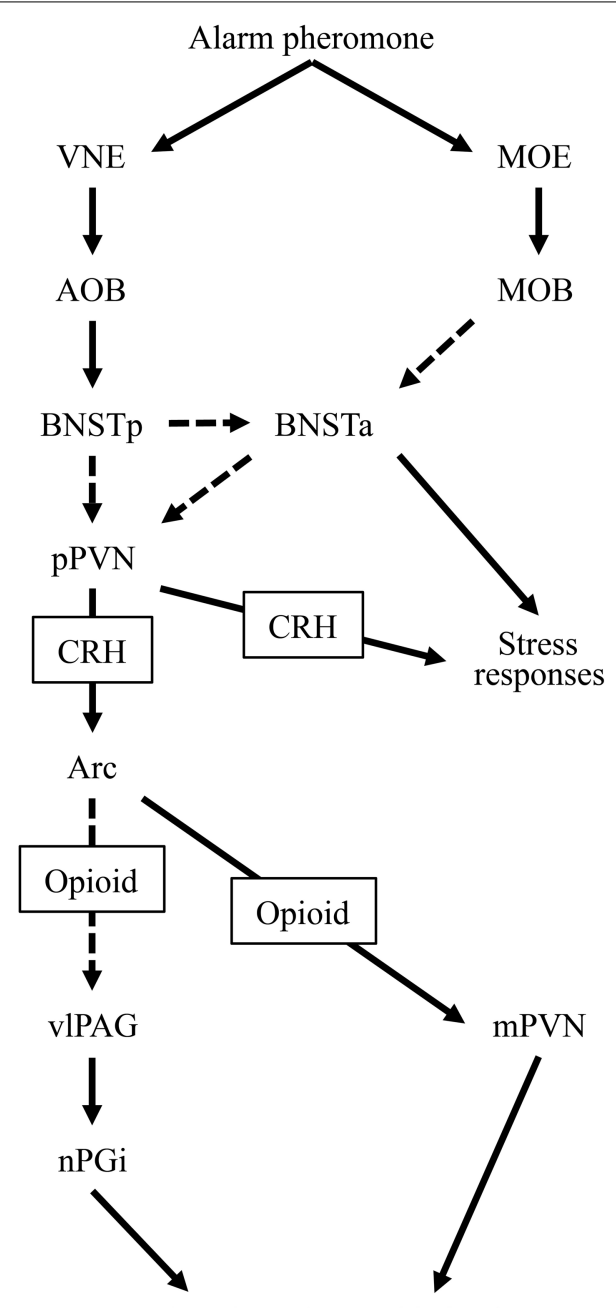

Deterioration of sexual behavior

FIGURE 4 | A diagram of the hypothesized neural circuit inducing deterioration in sexual behavior by the alarm pheromone. Solid arrows indicate direct projections while dotted arrows indicate indirect or unclear projections. Abbreviations: AOB, accessory olfactory bulb; Arc, arcuate nucleus; BNSTa, anterior division of the bed nucleus of the stria terminalis; BNSTp, posterior division of the bed nucleus of the stria terminalis; $\mathrm{CRH}$, corticotropin-releasing hormone; $\mathrm{MOB}$, main olfactory bulb; $\mathrm{MOE}$, main olfactory epithelium; mPVN, magnocellular part of the paraventricular nucleus of hypothalamus; nPGi, nucleus paragigantocellularis; PPVN, parvocellular part of the paraventricular nucleus of hypothalamus; VIPAG, ventrolateral periaqueductal gray; VNE, vomeronasal epithelium.

mPVN during deterioration in sexual behavior. Among these brain regions, the vlPAG and $\mathrm{mPVN}$ might be directly involved in the deterioration. It has been reported that lesioning the vlPAG reduces the number of mounts (Clark, 1975), suggesting that vlPAG activation deteriorates sexual behavior. The modulatory effects of the vlPAG might be exerted through the nPGi because lesioning the vlPAG (Clark, 1975) and nPGi (Yells et al., 1992) evokes the same pattern of deterioration. In addition, the vlPAG sends dense projections to the $\mathrm{nPGi}$, which are activated during sexual behavior (Normandin and Murphy, 2008). Therefore, the vlPAG and nPGi might compose a cluster that modulates sexual behavior. Similarly, the mPVN might be involved in the deterioration; it has been reported that lesions in both the mPVN and pPVN, but not in the pPVN, increase the number of mounts and decrease the hit rate (Liu et al., 1997a). Therefore, it has been suggested that suppression of the mPVN deteriorates male sexual behavior. However, the mPVN might deteriorate sexual behavior in parallel with the vlPAG-nPGi cluster because, to the best of our knowledge, the mPVN and vlPAG-nPGi cluster are not anatomically connected. In contrast, activation of the dlPAG seems to be less important for the deterioration of sexual behavior. Anatomical evidence suggests that this region receives sensory information regarding sexual behavior from genital organs in the female (Klop et al., 2005). This information might also activate the dIPAG of the male in the present study. The Arc might be indirectly involved in the deterioration as discussed below.

Based on the present findings, we suggest that naloxone attenuated, rather than compensated for, the alarm pheromoneinduced deterioration in sexual behavior. In the present and previous studies, rats that were pretreated with naloxone did not show a pheromone-induced deterioration in sexual behavior (Kobayashi et al., 2013b). One possible interpretation of this is that naloxone worked as an antagonist to the pheromone. Naloxone facilitates sexual behavior (McIntosh et al., 1980) and an opioid receptor antagonist increases Fos expression in nuclei that are not related to the nPGi, such as the nucleus accumbens core and shell, BNSTal, and central amygdala (Park and Carr, 1998; Carr et al., 1999). Therefore, an alternative interpretation could be that naloxone compensated the pheromone effects by facilitating sexual behavior through a separate neural mechanism. However, in the present study, we found that pretreatment with naloxone attenuated the pheromone-induced activation and suppression of the vlPAG-nPGi cluster and mPVN, respectively. Therefore, these results support the former interpretation.

Alarm pheromone-induced activation and suppression of the vlPAG-nPGi cluster and mPVN, respectively, were blocked by pretreatment with naloxone; therefore, these changes might be mediated by opioids. One possible source of opioids might be the Arc. Although its direct role in the deterioration of sexual behavior remains to be studied, the Arc contains abundant opioid peptides (Le Merrer et al., 2009), which are released by several stressors (Marinelli et al., 2004). In addition, the Arc sends direct projections to the mPVN (Sawchenko and Swanson, 1983); this suggests that opioid neurons in the Arc could directly suppress the mPVN. Indeed, an injection of opioids into the PVN inhibits male sexual behavior (Melis et al., 1999). The Arc could also affect the vlPAG-nPGi cluster because it sends projections to the medial preoptic area (MPOA) (Horvath et al., 1992). Electrical stimulation of the MPOA activates neurons in the vlPAG that project to the nPGi (Rizvi et al., 1996). Taken together, we propose that the Arc is the source of opioids that deteriorated the sexual behavior in the present study by suppressing the mPVN and activating the vlPAG-nPGi cluster.

In contrast to the suggested potential role of the MPOA, in our study, Fos expression in this region did not show any difference attributable to either the alarm pheromone or the pretreatment with naloxone. It is possible that the neural activation related to the expression of sexual behavior and/or intromissions obscured 
the alarm pheromone-induced difference in Fos expression. In this study, all the animals expressed 10 intromissions with equivalent latencies to mount, intromission, and tenth intromission, even when the alarm pheromone increased the number of mounts and decreased hit rate. It is known that the MPOA plays an important role in the expression of sexual behavior (Liu et al., 1997b). In addition, Fos expression in the MPOA is increased in subjects after 5 intromissions (Baum and Everitt, 1992). Therefore, high levels of Fos expression induced by the expression of sexual behavior and/or 10 intromissions might prevent us from observing any alarm pheromone-induced change of Fos expression in this region.

In the present study, we found that alarm pheromone-induced activation of the BNSTam, BNSTal, BNSTp, pPVN, and Arc was not blocked by pretreatment with naloxone. In the context of our hypothesized neural circuit that has been discussed above and in our previous study (Kobayashi et al., 2013a,b),these nuclei might be upstream of opioidergic neurons in the Arc. The alarm pheromone is perceived by the vomeronasal epithelium (Kiyokawa et al., 2007, 2013a), which transmits information to the accessory olfactory bulb, and subsequently, the BNSTp. This information is transmitted to the anterior BNST (BNSTa), composed of BNSTal and BNSTam, and the pPVN. It is currently unclear whether the information is transmitted from the BNSTp to the BNSTa and pPVN directly or indirectly. The BNSTa is only activated when it receives simultaneous information from the main olfactory system (Inagaki et al., 2014), which enhances the responsiveness of the pPVN to the stimuli (Inagaki et al., 2014). The activated pPVN sends CRHergic output to the Arc and induces opioid secretion. Therefore, pretreatment with naloxone may not affect the activity in these nuclei (Figure 4).

In summary, an alarm pheromone increased the number of mounts and decreased the hit rate in male rats. This was accompanied by an increase in Fos expression in the BNSTam, BNSTal, BNSTp, pPVN, Arc, dlPAG, vlPAG, and nPGi and a decrease in the $\mathrm{mPVN}$. Naloxone attenuated the pheromone-induced deterioration in sexual behavior and modification of Fos expression in the mPVN, vlPAG, and nPGi. Based on these results, we suggest that the alarm pheromone activates opioidergic neurons in the Arc, which deteriorate male sexual behavior by activating the vlPAG-nPGi cluster and suppressing the mPVN.

\section{ACKNOWLEDGMENTS}

This study was supported by JSPS KAKENHI Grant Numbers 21228006 and 23688035.

\section{REFERENCES}

Barrot, M., Wallace, D. L., Bolanos, C. A., Graham, D. L., Perrotti, L. I., Neve, R. L., et al. (2005). Regulation of anxiety and initiation of sexual behavior by CREB in the nucleus accumbens. Proc. Natl. Acad. Sci. U.S.A. 102, 8357-8362. doi: 10.1073/pnas.0500587102

Baum, M. J., and Everitt, B. J. (1992). Increased expression of c-fos in the medial preoptic area after mating in male rats: role of afferent inputs from the medial amygdala and midbrain central tegmental field. Neuroscience 50, 627-646. doi: 10.1016/0306-4522(92)90452-8

Carr, K. D., Kutchukhidze, N., and Park, T. H. (1999). Differential effects of mu and kappa opioid antagonists on Fos-like immunoreactivity in extended amygdala. Brain Res. 822, 34-42. doi: 10.1016/S0006-8993(99)01088-4

Clark, T. K. (1975). Sexual inhibition is reduced by rostral midbrain lesions in the male rat. Science 190, 169-171. doi: 10.1126/science.1166311
Horvath, T. L., Naftolin, F., and Leranth, C. (1992). GABAergic and catecholaminergic innervation of mediobasal hypothalamic beta-endorphin cells projecting to the medial preoptic area. Neuroscience 51, 391-399. doi: 10.1016/03064522(92)90323-T

Inagaki, H., Kiyokawa, Y., Kikusui, T., Takeuchi, Y., and Mori, Y. (2008). Enhancement of the acoustic startle reflex by an alarm pheromone in male rats. Physiol. Behav. 93, 606-611. doi: 10.1016/j.physbeh.2007.10.021

Inagaki, H., Kiyokawa, Y., Tamogami, S., Watanabe, H., Takeuchi, Y., and Mori, Y. (2014). Identification of a pheromone that increases anxiety in rats. Proc. Natl. Acad. Sci. U.S.A. 111, 18751-18756. doi: 10.1073/pnas.1414710112

Karlson, P., and Luscher, M. (1959). "Pheromones": a new term for a class of biologically active substances. Nature 183, 55-56. doi: 10.1038/183055a0

Kikusui, T., Takigami, S., Takeuchi, Y., and Mori, Y. (2001). Alarm pheromone enhances stress-induced hyperthermia in rats. Physiol. Behav. 72, 45-50. doi: 10.1016/S0031-9384(00)00370-X

Kiyokawa, Y., Honda, A., Takeuchi, Y., and Mori, Y. (2014). A familiar conspecific is more effective than an unfamiliar conspecific for social buffering of conditioned fear responses in male rats. Behav. Brain Res. 267, 189-193. doi: 10.1016/j.bbr.2014.03.043

Kiyokawa, Y., Kikusui, T., Takeuchi, Y., and Mori, Y. (2004). Alarm pheromones with different functions are released from different regions of the body surface of male rats. Chem. Senses 29, 35-40. doi: 10.1093/chemse/bjh004

Kiyokawa, Y., Kikusui, T., Takeuchi, Y., and Mori, Y. (2005a). Alarm pheromone that aggravates stress-induced hyperthermia is soluble in water. Chem. Senses 30, 513-519. doi: 10.1093/chemse/bji044

Kiyokawa, Y., Kikusui, T., Takeuchi, Y., and Mori, Y. (2005b). Mapping the neural circuit activated by alarm pheromone perception by c-Fos immunohistochemistry. Brain Res. 1043, 145-154. doi: 10.1016/j.brainres.2005.02.061

Kiyokawa, Y., Kikusui, T., Takeuchi, Y., and Mori, Y. (2007). Removal of the vomeronasal organ blocks the stress-induced hyperthermia response to alarm pheromone in male rats. Chem. Senses 32, 57-64. doi: 10.1093/chemse/bjl036

Kiyokawa, Y., Kodama, Y., Kubota, T., Takeuchi, Y., and Mori, Y. (2013a). Alarm pheromone is detected by the vomeronasal organ in male rats. Chem. Senses 38, 661-668. doi: 10.1093/chemse/bjt030

Kiyokawa, Y., Kodama, Y., Takeuchi, Y., and Mori, Y. (2013b). Physical interaction is not necessary for the induction of housing-type social buffering of conditioned hyperthermia in male rats. Behav. Brain Res. 256, 414-419. doi: 10.1016/j.bbr.2013.08.037

Kiyokawa, Y., Shimozuru, M., Kikusui, T., Takeuchi, Y., and Mori, Y. (2006). Alarm pheromone increases defensive and risk assessment behaviors in male rats. Physiol. Behav. 87, 383-387. doi: 10.1016/j.physbeh.2005.11.003

Klein, S. L. (2003). Parasite manipulation of the proximate mechanisms that mediate social behavior in vertebrates. Physiol. Behav. 79, 441-449. doi: 10.1016/S0031-9384(03)00163-X

Klop, E. M., Mouton, L. J., Kuipers, R., and Holstege, G. (2005). Neurons in the lateral sacral cord of the cat project to periaqueductal grey, but not to thalamus. Eur. J. Neurosci. 21, 2159-2166. doi: 10.1111/j.1460-9568.2005.04039.x

Kobayashi, T., Kiyokawa, Y., Arata, S., Takeuchi, Y., and Mori, Y. (2013a). c-Fos expression during the modulation of sexual behavior by an alarm pheromone. Behav. Brain Res. 237, 230-237. doi: 10.1016/j.bbr.2012.09.042

Kobayashi, T., Kiyokawa, Y., Arata, S., Takeuchi, Y., and Mori, Y. (2013b). Naloxone blocks the deterioration of male sexual behavior induced by alarm pheromone in rats. J. Vet. Med. Sci. 75, 761-765. doi: 10.1292/jvms.12-0454

Kobayashi, T., Kiyokawa, Y., Takeuchi, Y., and Mori, Y. (2011). Pretreatment with CP-154526 blocks the modifying effects of alarm pheromone on components of sexual behavior in male, but not in female, rats. Chem. Senses 36, 623-632. doi: 10.1093/chemse/bjr017

Le Merrer, J., Becker, J. A., Befort, K., and Kieffer, B. L. (2009). Reward processing by the opioid system in the brain. Physiol. Rev. 89, 1379-1412. doi: 10.1152/physrev.00005.2009

Lin, Y. C., Rikihisa, Y., Kono, H., and Gu, Y. (1990). Effects of larval tapeworm (Taenia taeniaeformis) infection on reproductive functions in male and female host rats. Exp. Parasitol. 70, 344-352. doi: 10.1016/0014-4894(90)90116-T

Liu, Y. C., Salamone, J. D., and Sachs, B. D. (1997a). Impaired sexual response after lesions of the paraventricular nucleus of the hypothalamus in male rats. Behav. Neurosci. 111, 1361-1367. doi: 10.1037/0735-7044.111.6.1361

Liu, Y. C., Salamone, J. D., and Sachs, B. D. (1997b). Lesions in medial preoptic area and bed nucleus of stria terminalis: differential effects on copulatory behavior and noncontact erection in male rats. J. Neurosci. 17, 5245-5253. 
Marinelli, P. W., Quirion, R., and Gianoulakis, C. (2004). An in vivo profile of beta-endorphin release in the arcuate nucleus and nucleus accumbens following exposure to stress or alcohol. Neuroscience 127, 777-784. doi: 10.1016/j.neuroscience.2004.05.047

McIntosh, T. K., Vallano, M. L., and Barfield, R. J. (1980). Effects of morphine, beta-endorphin and naloxone on catecholamine levels and sexual behavior in the male rat. Pharmacol. Biochem. Behav. 13, 435-441. doi: 10.1016/00913057(80)90251-8

Melis, M. R., Succu, S., Spano, M. S., and Argiolas, A. (1999). Morphine injected into the paraventricular nucleus of the hypothalamus prevents noncontact penile erections and impairs copulation: involvement of nitric oxide. Eur. J. Neurosci. 11, 1857-1864. doi: 10.1046/j.1460-9568.1999.00603.x

Normandin, J. J., and Murphy, A. Z. (2008). Nucleus paragigantocellularis afferents in male and female rats: organization, gonadal steroid receptor expression, and activation during sexual behavior. J. Comp. Neurol. 508, 771-794. doi: $10.1002 / \mathrm{cne} .21704$

Park, T. H., and Carr, K. D. (1998). Neuroanatomical patterns of fos-like immunoreactivity induced by a palatable meal and meal-paired environment in saline- and naltrexone-treated rats. Brain Res. 805, 169-180. doi: 10.1016/S0006-8993(98)00719-7

Paxinos, G., and Watson, C. (2007). The Rat Brain in Stereotaxic Coordinates. Tokyo: Elsevier.

Retana-Marquez, S., Salazar, E. D., and Velazquez-Moctezuma, J. (1996). Effect of acute and chronic stress on masculine sexual behavior in the rat. Psychoneuroendocrinology 21, 39-50. doi: 10.1016/0306-4530(95) 00029-1

Rhees, R. W., Lephart, E. D., and Eliason, D. (2001). Effects of maternal separation during early postnatal development on male sexual behavior and female reproductive function. Behav. Brain Res. 123, 1-10. doi: 10.1016/S01664328(00)00381-8
Rizvi, T. A., Murphy, A. Z., Ennis, M., Behbehani, M. M., and Shipley, M. T. (1996). Medial preoptic area afferents to periaqueductal gray medullo-output neurons: a combined Fos and tract tracing study. J. Neurosci. 16, 333-344.

Sawchenko, P. E., and Swanson, L. W. (1983). The organization of forebrain afferents to the paraventricular and supraoptic nuclei of the rat. J. Comp. Neurol. 218, 121-144. doi: 10.1002/cne.902180202

Wang, C. T., Shui, H. A., Huang, R. L., Tai, M. Y., Peng, M. T., and Tsai, Y. F. (2006). Sexual motivation is demasculinized, but not feminized, in prenatally stressed male rats. Neuroscience 138, 357-364. doi: 10.1016/j.neuroscience.2005.11.026

Yells, D. P., Hendricks, S. E., and Prendergast, M. A. (1992). Lesions of the nucleus paragigantocellularis: effects on mating behavior in male rats. Brain Res. 596, 73-79. doi: 10.1016/0006-8993(92)91534-L

Conflict of Interest Statement: The authors declare that the research was conducted in the absence of any commercial or financial relationships that could be construed as a potential conflict of interest.

Received: 14 November 2014; accepted: 06 February 2015; published online: 23 February 2015.

Citation: Kobayashi T, Kiyokawa Y, Takeuchi Y and Mori Y (2015) Neural correlates underlying naloxone-induced amelioration of sexual behavior deterioration due to an alarm pheromone. Front. Neurosci. 9:52. doi: 10.3389/fnins.2015.00052

This article was submitted to Systems Biology, a section of the journal Frontiers in Neuroscience.

Copyright (c) 2015 Kobayashi, Kiyokawa, Takeuchi and Mori. This is an open-access article distributed under the terms of the Creative Commons Attribution License (CC BY). The use, distribution or reproduction in other forums is permitted, provided the original author(s) or licensor are credited and that the original publication in this journal is cited, in accordance with accepted academic practice. No use, distribution or reproduction is permitted which does not comply with these terms. 